\title{
Measurement and Correlation of the Solubility of Maltitol in Different Pure Solvents, Methanol-Water Mixtures and Ethanol-Water Mixtures
}

Zhenfang $\mathrm{Li}^{a, b}$, Teng Zhang ${ }^{a, b}$, Cui Huang ${ }^{a, b}$, Haisheng Wang ${ }^{a, b}$, Bo Yu ${ }^{a, b}$, Junbo $\operatorname{Gong}^{a, b, *}$

${ }^{a}$ School of Chemical Engineering and Technology, State Key Laboratory of Chemical Engineering, Tianjin University, Tianjin 300072, People’s Republic of China

${ }^{b}$ The Co-Innovation Center of Chemistry and Chemical Engineering of Tianjin, Tianjin University

\section{AUTHOR INFORMATION}

\section{Corresponding Author}

Tel.: 86-22-27405754. Fax: +86-22-27374971. E-mail: junbo_gong@tju.edu.cn. 
Table S1. Parameters of the Van't Hoff Equation for Maltitol in Pure Solvents

\begin{tabular}{ccccc}
\hline solvent & $\Delta H_{d}$ & $\Delta S_{d}$ & $A R D \%$ & $R M S D \cdot 10^{-4}$ \\
\hline water & 24344 & 58 & 2.6026 & 21.8136 \\
DMF & 24190 & 40 & 3.9464 & 3.5099 \\
methanol & 29715 & 31 & 3.9249 & 0.1923 \\
ethanol & 30930 & 18 & 2.7046 & 0.0189 \\
2-propanol & 32287 & 17 & 5.6927 & 0.0194 \\
\hline
\end{tabular}

Table S2. Parameters of the Modified Apelblat Equation for Maltitol in Pure Solvents

\begin{tabular}{cccccc}
\hline solvent & $A$ & $B$ & $C$ & $A R D \%$ & $R M S D \cdot 10^{-4}$ \\
\hline water & -214.4 & 7288.7 & 32.8 & 2.5504 & 23.4868 \\
DMF & -748 & 31911 & 112 & 0.9851 & 1.0739 \\
methanol & -745 & 31109 & 111 & 1.4306 & 0.0691 \\
ethanol & -398 & 14824 & 59 & 1.655 & 0.0117 \\
2-propanol & -945 & 40002 & 140 & 3.2404 & 0.0103 \\
\hline
\end{tabular}

Table S3. Parameters of the Equation 5 for Maltitol in Binary Solvents

\begin{tabular}{lccccc}
\hline$x_{B}^{0}$ & $A$ & $B$ & $C$ & $A R D \%$ & $R M S D \cdot 10^{-4}$ \\
\hline \multicolumn{7}{c}{ binary methanol-water mixtures } \\
0.0588 & -47.6 & -1270.7 & 8.5 & 1.5834 & 12.0000 \\
0.1232 & 242 & -14564 & -35 & 1.5815 & 9.0313 \\
\hline
\end{tabular}




\begin{tabular}{rccccc}
\hline 0.1942 & -150.9 & 3046.3 & 24 & 2.5441 & 14.0000 \\
0.2726 & -1190 & 50185 & 179 & 1.7319 & 5.6926 \\
0.3599 & -1338 & 57150 & 201 & 1.9005 & 4.8688 \\
0.4575 & -1462 & 62145 & 219 & 2.5003 & 7.2399 \\
0.5675 & -725 & 27584 & 110 & 2.6171 & 2.7335 \\
0.6922 & -1021 & 41523 & 154 & 1.7247 & 0.8678 \\
0.8350 & -935 & 37162 & 141 & 2.7274 & 0.4892 \\
\hline \multicolumn{7}{c}{ binary ethanol-water mixtures } \\
0.0416 & -170.1 & 4303.6 & 26.7 & 2.9060 & 22.0000 \\
0.0891 & 226 & -13974 & -32 & 2.6502 & 20.0000 \\
0.1435 & -96.9 & 720.7 & 16.0 & 2.7622 & 19.0000 \\
0.2068 & -352 & 12467 & 54 & 2.1887 & 13.0000 \\
0.2811 & -1236 & 52894 & 185 & 2.2869 & 15.0000 \\
0.3697 & -424 & 15295 & 65 & 2.2194 & 6.3495 \\
0.4771 & -1512 & 64336 & 227 & 2.5643 & 5.3276 \\
0.6100 & 96 & -8962.7 & -12.7 & 2.2701 & 0.8518 \\
0.7787 & 261 & -16142 & -38 & 2.4287 & 0.1620 \\
\hline
\end{tabular}

Table S4. Parameters of the Equation 7 for Maltitol in Binary Solvents

\begin{tabular}{cccccccc}
\hline \multirow{2}{*}{$T / K$} & $B_{0}$ & $B_{1}$ & $B_{2}$ & $B_{3}$ & $B_{4}$ & \multirow{2}{*}{$A R D \%$} & $R M S D$ \\
\hline \multicolumn{7}{c}{ binary methanol-water mixtures } \\
298.15 & -2.6530 & -9.0958 & 23.3840 & 40.2526 & 20.5121 & 3.7564 & 0.0009 \\
303.15 & -2.5654 & -7.4840 & 16.4172 & 27.9751 & 13.3402 & 5.2736 & 0.0011 \\
308.15 & -2.2778 & -7.6177 & 14.5238 & 22.2443 & 9.6838 & 5.1068 & 0.0018 \\
313.15 & -2.0168 & -9.3883 & 25.6697 & 42.3297 & 20.8046 & 5.7259 & 0.0020 \\
318.15 & -1.8568 & -9.3775 & 29.5782 & 50.8939 & 25.8345 & 3.1241 & 0.0013 \\
323.15 & -1.6842 & -9.3265 & 32.0172 & 56.0761 & 29.0075 & 3.2108 & 0.0016 \\
\hline \multicolumn{7}{c}{ binary ethanol-water mixtures } \\
298.15 & -3.2351 & -1.0903 & 3.0162 & 33.9504 & 27.3714 & 2.6192 & 0.0008 \\
303.15 & -3.1134 & 0.0295 & -3.4174 & 19.7943 & 17.7221 & 1.9818 & 0.0006 \\
308.15 & -2.8585 & -0.7578 & -0.4880 & 23.0540 & 18.6566 & 1.4274 & 0.0010 \\
313.15 & -2.6505 & 0.5819 & -8.4448 & -6.7267 & 8.2913 & 0.9164 & 0.0008 \\
318.15 & -2.3052 & -2.7890 & 13.7353 & 51.2584 & 35.4929 & 3.2673 & 0.0014 \\
323.15 & -2.1821 & -2.6640 & 14.2377 & 50.5689 & 33.6790 & 3.8179 & 0.0019 \\
\hline \multicolumn{7}{c}{}
\end{tabular}

Table S5. Parameters of the Equation 12 for Maltitol in Binary Solvents

\begin{tabular}{ccccccc}
\hline solvent & $A_{1}$ & $A_{2}$ & $A_{3}$ & $A_{4}$ & $A_{5}$ & $A_{6}$ \\
\hline Methanol-water & 197.429 & 5770.408 & 30.78586 & 1332.32 & 56757.13 & 7166.207 \\
\hline
\end{tabular}




\begin{tabular}{ccccccc}
\hline Ethanol-water & 377.849 & 13542.71 & 57.7699 & 88.35621 & -3828.8 & 1048.943 \\
\hline solvent & \multirow{2}{*}{$A_{7}$} & \multirow{2}{*}{$A_{8}$} & \multicolumn{1}{c}{$A_{9}$} & \multirow{2}{*}{ ARD\% } & \multirow{2}{*}{ RMSD } & \\
& & & & & & \\
\hline Methanol-water & -12173.5 & 6047.489 & 198.822 & 5.5694 & 0.0022 & \\
Ethanol-water & -9759.2 & 7411.458 & -13.4498 & 5.1245 & 0.0025 & \\
\hline
\end{tabular}

Table S6. Parameters of the Equation 12 for Maltitol in Binary Solvents

\begin{tabular}{cccccc}
\hline solvent & $C_{1}$ & $C_{2}$ & $C_{3}$ & $C_{4}$ & $C_{5}$ \\
\hline Methanol-water & 10.00312 & -3782.23 & 7.32497 & -4935.95 & 7166.207 \\
\hline Ethanol-water & 11.39817 & -4382.89 & -2.26718 & 344.5836 & 1048.943 \\
\hline solvent & $C_{6}$ & $C_{7}$ & $A R D \%$ & $R M S D$ & \\
\hline Methanol-water & -12173.5 & 6047.488 & 6.3348 & 0.0022 & \\
Ethanol-water & -9759.2 & 7411.458 & 5.1440 & 0.0022 & \\
\hline
\end{tabular}

Table S7. The Percent Deviation $(P D)$ of the Measured and Predicted Solubility Values of Maltitol in Pure Solvents

\begin{tabular}{|c|c|c|c|}
\hline \multirow[b]{2}{*}{$T / \mathrm{K}$} & \multirow[b]{2}{*}{$10^{2} x_{A}^{e x p}$} & \multicolumn{2}{|c|}{$10^{2} P D$} \\
\hline & & Apelblat & Van't Hoff \\
\hline \multicolumn{4}{|c|}{ water } \\
\hline 298.15 & 6.1564 & 2.39 & 3.96 \\
\hline 303.15 & 6.7109 & 3.32 & 3.59 \\
\hline 308.15 & 7.8555 & 2.27 & 3.51 \\
\hline 313.15 & 9.7233 & 3.90 & 2.67 \\
\hline 318.15 & 11.0805 & 1.58 & 1.07 \\
\hline 323.15 & 12.5382 & 1.84 & 0.81 \\
\hline \multicolumn{4}{|c|}{ DMF } \\
\hline 298.15 & 0.7610 & 0.83 & 7.81 \\
\hline 303.15 & 0.8187 & 1.07 & 0.67 \\
\hline 308.15 & 0.9216 & 1.13 & 4.49 \\
\hline 313.15 & 1.0931 & 1.53 & 2.43 \\
\hline 318.15 & 1.2811 & 0.64 & 1.15 \\
\hline 323.15 & 1.5273 & 0.73 & 2.27 \\
\hline \multicolumn{4}{|c|}{ methanol } \\
\hline 298.15 & 0.0290 & 1.03 & 7.24 \\
\hline 303.15 & 0.0337 & 1.19 & 2.67 \\
\hline 308.15 & 0.0393 & 1.53 & 1.27 \\
\hline 313.15 & 0.0453 & 1.55 & 5.74 \\
\hline 318.15 & 0.0552 & 1.63 & 3.80 \\
\hline 323.15 & 0.0706 & 1.27 & 3.54 \\
\hline
\end{tabular}




\begin{tabular}{|c|c|c|c|}
\hline \multicolumn{4}{|c|}{ ethanol } \\
\hline 298.15 & 0.0036 & 1.42 & 3.47 \\
\hline 303.15 & 0.0044 & 2.07 & 2.97 \\
\hline 308.15 & 0.0052 & 1.18 & 0.18 \\
\hline 313.15 & 0.0062 & 0.88 & 2.99 \\
\hline 318.15 & 0.0074 & 2.57 & 3.85 \\
\hline 323.15 & 0.0094 & 1.80 & 2.76 \\
\hline \multicolumn{4}{|c|}{ 2-propanol } \\
\hline 298.15 & 0.0019 & 2.68 & 8.29 \\
\hline 303.15 & 0.0023 & 3.84 & 5.53 \\
\hline 308.15 & 0.0027 & 4.10 & 0.65 \\
\hline 313.15 & 0.0030 & 5.52 & 10.97 \\
\hline 318.15 & 0.0038 & 1.15 & 4.20 \\
\hline 323.15 & 0.0051 & 2.16 & 4.53 \\
\hline
\end{tabular}

Table S8. The Percent Deviation (PD) of the Measured and Predicted Solubility Values of Maltitol in Methanol-Water Mixtures

\begin{tabular}{|c|c|c|c|c|c|}
\hline \multirow[b]{2}{*}{$x_{B}^{0}$} & \multirow[b]{2}{*}{$10^{2} x_{A}^{e x p}$} & \multicolumn{4}{|c|}{$10^{2} P D$} \\
\hline & & eq 5 & eq 7 & eq 12 & eq 15 \\
\hline \multicolumn{6}{|c|}{$T=298.15 \mathrm{~K}$} \\
\hline 0.0588 & 4.3265 & 1.41 & 2.58 & 0.84 & 0.98 \\
\hline 0.1232 & 3.2424 & 1.63 & 5.88 & 7.15 & 9.33 \\
\hline 0.1942 & 2.2760 & 0.57 & 2.01 & 2.58 & 5.44 \\
\hline 0.2726 & 1.4967 & 0.71 & 11.05 & 11.69 & 7.68 \\
\hline 0.3599 & 1.3170 & 1.20 & 9.54 & 7.74 & 11.71 \\
\hline 0.4575 & 0.7657 & 1.89 & 0.38 & 2.89 & 2.35 \\
\hline 0.5675 & 0.3956 & 1.16 & 1.97 & 5.74 & 0.58 \\
\hline 0.6922 & 0.1691 & 0.35 & 0.35 & 1.42 & 5.68 \\
\hline 0.8350 & 0.0607 & 0.66 & 0.00 & 4.94 & 12.69 \\
\hline \multicolumn{6}{|c|}{$T=303.15 \mathrm{~K}$} \\
\hline 0.0588 & 5.0883 & 3.67 & 2.43 & 4.87 & 5.34 \\
\hline 0.1232 & 3.9218 & 2.53 & 4.79 & 5.44 & 4.89 \\
\hline 0.1942 & 2.8484 & 1.25 & 2.67 & 3.52 & 2.81 \\
\hline 0.2726 & 1.8855 & 2.99 & 9.66 & 10.54 & 11.53 \\
\hline 0.3599 & 1.6457 & 3.71 & 10.03 & 7.33 & 6.33 \\
\hline 0.4575 & 0.9012 & 2.45 & 6.35 & 10.69 & 12.11 \\
\hline 0.5675 & 0.5627 & 3.61 & 7.14 & 4.71 & 3.27 \\
\hline 0.6922 & 0.2174 & 0.92 & 3.63 & 2.81 & 4.65 \\
\hline 0.8350 & 0.0776 & 3.74 & 0.77 & 1.03 & 1.03 \\
\hline \multicolumn{6}{|c|}{$T=308.15 \mathrm{~K}$} \\
\hline 0.0588 & 6.6848 & 2.86 & 2.58 & 1.90 & 0.41 \\
\hline 0.1232 & 4.9394 & 0.92 & 2.71 & 6.77 & 4.93 \\
\hline 0.1942 & 3.8718 & 6.26 & 10.15 & 11.00 & 8.78 \\
\hline 0.2726 & 2.2370 & 3.43 & 13.62 & 17.99 & 21.60 \\
\hline
\end{tabular}




\begin{tabular}{|c|c|c|c|c|c|}
\hline 0.3599 & 1.9255 & 2.67 & 6.07 & 1.42 & 5.17 \\
\hline 0.4575 & 1.1816 & 1.17 & 0.95 & 9.58 & 14.41 \\
\hline 0.5675 & 0.7205 & 4.04 & 5.00 & 1.68 & 3.46 \\
\hline 0.6922 & 0.3074 & 4.62 & 3.94 & 1.72 & 4.33 \\
\hline 0.8350 & 0.1177 & 6.03 & 1.02 & 9.43 & 2.89 \\
\hline \multicolumn{6}{|c|}{$T=313.15 \mathrm{~K}$} \\
\hline 0.0588 & 7.9945 & 0.45 & 3.87 & 1.26 & 2.72 \\
\hline 0.1232 & 6.2105 & 2.06 & 7.64 & 7.24 & 5.50 \\
\hline 0.1942 & 4.3930 & 3.87 & 2.67 & 0.57 & 1.78 \\
\hline 0.2726 & 3.0522 & 0.32 & 8.12 & 11.11 & 14.34 \\
\hline 0.3599 & 2.5531 & 1.04 & 2.67 & 0.21 & 3.29 \\
\hline 0.4575 & 1.6139 & 0.66 & 4.63 & 6.59 & 11.05 \\
\hline 0.5675 & 1.0944 & 3.60 & 13.16 & 12.02 & 7.67 \\
\hline 0.6922 & 0.3896 & 3.70 & 7.11 & 8.44 & 14.76 \\
\hline 0.8350 & 0.1546 & 0.91 & 1.62 & 0.26 & 6.53 \\
\hline \multicolumn{6}{|c|}{$T=318.15 \mathrm{~K}$} \\
\hline 0.0588 & 9.6212 & 0.96 & 2.55 & 4.29 & 4.59 \\
\hline 0.1232 & 7.3974 & 1.15 & 4.74 & 1.91 & 1.53 \\
\hline 0.1942 & 5.6375 & 1.64 & 2.24 & 0.81 & 0.34 \\
\hline 0.2726 & 4.2335 & 2.01 & 6.04 & 4.33 & 4.93 \\
\hline 0.3599 & 3.5867 & 2.13 & 1.17 & 5.71 & 5.05 \\
\hline 0.4575 & 2.4541 & 5.86 & 0.95 & 4.83 & 4.04 \\
\hline 0.5675 & 1.4745 & 2.41 & 5.62 & 8.88 & 7.98 \\
\hline 0.6922 & 0.5725 & 0.02 & 3.91 & 6.53 & 7.76 \\
\hline 0.8350 & 0.2185 & 3.20 & 0.92 & 6.00 & 7.41 \\
\hline \multicolumn{6}{|c|}{$T=323.15 \mathrm{~K}$} \\
\hline 0.0588 & 11.8244 & 0.16 & 0.20 & 5.56 & 3.63 \\
\hline 0.1232 & 8.5645 & 1.21 & 1.20 & 7.40 & 4.85 \\
\hline 0.1942 & 7.3101 & 1.69 & 4.06 & 1.17 & 4.10 \\
\hline 0.2726 & 5.7967 & 0.92 & 2.49 & 0.47 & 3.17 \\
\hline 0.3599 & 4.9350 & 0.66 & 1.23 & 7.56 & 11.58 \\
\hline 0.4575 & 3.3289 & 2.96 & 5.53 & 2.81 & 7.83 \\
\hline 0.5675 & 2.2150 & 0.88 & 9.13 & 13.24 & 18.49 \\
\hline 0.6922 & 0.8398 & 0.75 & 4.24 & 7.98 & 0.36 \\
\hline 0.8350 & 0.3394 & 1.71 & 0.82 & 6.19 & 2.53 \\
\hline
\end{tabular}

Table S9. The Percent Deviation $(P D)$ of the Measured and Predicted Solubility Values of Maltitol in Ethanol-Water Mixtures

\begin{tabular}{llllll}
\hline & & \multicolumn{5}{c}{$10^{2} x_{A}^{\text {cal }}$} \\
\cline { 3 - 6 }$x_{B}^{0}$ & $10^{2} x_{A}^{\text {exp }}$ & eq 5 & eq 7 & eq 12 & eq 15 \\
\hline & \multicolumn{6}{c}{$T=298.15 \mathrm{~K}$} \\
0.0416 & 3.8364 & 2.61 & 1.69 & 5.77 & 8.05 \\
0.0891 & 3.5543 & 2.06 & 0.65 & 3.29 & 5.61 \\
0.1435 & 3.1113 & 1.62 & 5.33 & 1.89 & 0.52 \\
\hline
\end{tabular}




\begin{tabular}{|c|c|c|c|c|c|}
\hline 0.2068 & 2.8916 & 1.67 & 3.76 & 5.68 & 7.88 \\
\hline 0.2811 & 2.1741 & 1.21 & 5.63 & 5.51 & 7.68 \\
\hline 0.3697 & 1.1372 & 0.77 & 4.76 & 8.05 & 5.64 \\
\hline 0.4771 & 0.4794 & 0.96 & 0.29 & 6.61 & 4.30 \\
\hline 0.6100 & 0.1253 & 0.32 & 1.20 & 4.55 & 2.39 \\
\hline 0.7787 & 0.0268 & 1.87 & 0.37 & 10.07 & 11.94 \\
\hline \multicolumn{6}{|c|}{$T=303.15 \mathrm{~K}$} \\
\hline 0.0416 & 4.4390 & 3.48 & 0.48 & 0.58 & 1.18 \\
\hline 0.0891 & 4.3281 & 2.32 & 1.07 & 1.98 & 1.39 \\
\hline 0.1435 & 3.8022 & 0.88 & 3.97 & 2.87 & 3.47 \\
\hline 0.2068 & 3.4252 & 1.85 & 2.18 & 1.70 & 1.13 \\
\hline 0.2811 & 2.4721 & 1.08 & 0.41 & 2.84 & 3.42 \\
\hline 0.3697 & 1.4843 & 2.90 & 2.82 & 3.05 & 3.62 \\
\hline 0.4771 & 0.5793 & 1.12 & 4.33 & 11.07 & 11.65 \\
\hline 0.6100 & 0.1689 & 1.12 & 2.25 & 0.53 & 0.00 \\
\hline 0.7787 & 0.0337 & 1.78 & 0.30 & 6.23 & 5.64 \\
\hline \multicolumn{6}{|c|}{$T=308.15 \mathrm{~K}$} \\
\hline 0.0416 & 5.5278 & 2.21 & 0.29 & 0.59 & 2.65 \\
\hline 0.0891 & 5.3913 & 2.80 & 2.43 & 2.07 & 0.09 \\
\hline 0.1435 & 4.5770 & 4.70 & 4.77 & 6.30 & 8.42 \\
\hline 0.2068 & 4.2121 & 2.41 & 3.79 & 0.54 & 1.42 \\
\hline 0.2811 & 2.9951 & 1.49 & 0.25 & 5.83 & 7.88 \\
\hline 0.3697 & 1.7871 & 2.43 & 0.28 & 7.28 & 9.31 \\
\hline 0.4771 & 0.7646 & 0.24 & 0.54 & 6.58 & 8.54 \\
\hline 0.6100 & 0.2138 & 2.48 & 0.42 & 1.26 & 3.04 \\
\hline 0.7787 & 0.0422 & 3.79 & 0.00 & 1.18 & 0.47 \\
\hline \multicolumn{6}{|c|}{$T=313.15 \mathrm{~K}$} \\
\hline 0.0416 & 7.1213 & 2.37 & 0.07 & 1.99 & 0.08 \\
\hline 0.0891 & 6.9822 & 2.18 & 0.80 & 5.17 & 3.34 \\
\hline 0.1435 & 6.2065 & 3.82 & 2.25 & 1.76 & 0.10 \\
\hline 0.2068 & 5.4728 & 1.84 & 2.98 & 4.06 & 2.27 \\
\hline 0.2811 & 3.8053 & 1.31 & 1.70 & 4.59 & 6.50 \\
\hline 0.3697 & 2.3040 & 1.84 & 0.41 & 4.98 & 6.86 \\
\hline 0.4771 & 1.0093 & 3.49 & 0.02 & 2.86 & 4.66 \\
\hline 0.6100 & 0.2977 & 4.64 & 0.00 & 5.84 & 4.23 \\
\hline 0.7787 & 0.0584 & 5.82 & 0.00 & 5.82 & 4.28 \\
\hline \multicolumn{6}{|c|}{$T=318.15 \mathrm{~K}$} \\
\hline 0.0416 & 8.9119 & 3.99 & 1.68 & 0.98 & 0.59 \\
\hline 0.0891 & 8.6343 & 3.93 & 2.88 & 3.14 & 2.77 \\
\hline 0.1435 & 7.6533 & 3.13 & 1.10 & 0.54 & 0.92 \\
\hline 0.2068 & 6.9572 & 3.20 & 1.74 & 4.77 & 4.41 \\
\hline 0.2811 & 5.4018 & 5.89 & 0.24 & 6.90 & 6.56 \\
\hline 0.3697 & 3.1574 & 3.93 & 7.18 & 2.78 & 2.43 \\
\hline 0.4771 & 1.6091 & 6.85 & 10.20 & 17.37 & 17.08 \\
\hline
\end{tabular}




\begin{tabular}{rrrrrr}
0.6100 & 0.3505 & 3.82 & 3.82 & 4.02 & 4.37 \\
0.7787 & 0.0678 & 0.15 & 0.44 & 7.67 & 7.96 \\
0.0416 & 10.2478 & 2.76 & 0.62 & 9.60 & 6.91 \\
0.0891 & 9.6836 & 2.61 & 0.53 & 9.79 & 7.12 \\
0.1435 & 8.9654 & 2.42 & 0.54 & 9.00 & 6.39 \\
0.2068 & 8.3145 & 2.16 & 2.23 & 1.17 & 1.22 \\
0.2811 & 6.7661 & 2.75 & 2.53 & 5.53 & 7.72 \\
0.3697 & 3.8957 & 1.44 & 10.32 & 0.55 & 1.73 \\
0.4771 & 2.1878 & 2.74 & 12.78 & 21.79 & 23.51 \\
0.6100 & 0.4675 & 1.33 & 4.19 & 1.84 & 0.34 \\
0.7787 & 0.0815 & 1.23 & 0.49 & 19.02 & 16.56 \\
\hline
\end{tabular}

